\title{
A Second Order Finite Difference Approximation for the Fractional Diffusion Equation
}

\author{
H. M. Nasir, Member, IACSIT, B. L. K. Gunawardana, and H. M. N. P. Abeyrathna
}

\begin{abstract}
We consider an approximation of one-dimensional fractional diffusion equation. We claim and show that the finite difference approximation obtained from the Grünwald-Letnikov formulation, often claimed to be of first order accuracy, is in fact a second order approximation of the fractional derivative at a point away from the grid points. We use this fact to device a second order accurate finite difference approximation for the fractional diffusion equation. The proposed method is also shown to be unconditionally stable. By this approach, we treat three cases of difference approximations in a unified setting. The results obtained are justified by numerical examples.
\end{abstract}

Index Terms-Fractional derivative, diffusion equation, grunwald approximation, crank-nicolson method.

\section{INTRODUCTION}

Fractional derivative is becoming a popular tool to model various physical phenomena and to describe the dynamical characteristics of the physical system better than the standard integer order derivatives. For example, the reaction-diffusion problem is better described by fractional differential equation than the classical diffusion equation when there is a presence of anomalous diffusion of particles [1], [2]. There are some probabilistic interpretations of the fractional derivatives that better suits in the control theory as well [3], [4].

Consider the numerical approximation of the one-dimensional fractional diffusion equation

$$
\frac{\partial}{\partial t} u(x, t)=d(x) \frac{\partial^{\alpha}}{\partial x^{\alpha}} u(x, t)+q(x, t)
$$

defined in a bounded spatial domain $x_{L}<x<x_{R}$ with time domain $t>0$. Here, $d(x)>0$ is the diffusion coefficient and $q(x, t)$ is the forcing term representing either a source or sink. The initial and boundary conditions are given by

$$
u\left(x_{L}, t\right)=0 ; u\left(x_{R}, t\right)=b_{R}(t), t>0 ; \quad u(x, 0)=s_{0}(x) .
$$

We assume that $1 \leq \alpha \leq 2$ which is considered useful for applications. The fractional derivative in the (1) is a (left-sided) Riemann-Liouville derivative of order $\alpha$ defined as

Manuscript received February 5, 2013; revised May 17, 2013.

H. M. Nasir is with the Sultan Qaboos University, Al-khoud 123, Muscat, Oman (e-mail: nasirh@squ.edu.om,nasirhm11@yahoo.com).

B. L. K. Gunawardana and H. M. N. P. Abeyrathna are with University of Peradeniya, Sri lanka (e-mail: leksh.gg@gmail.com,npa512@yahoo.com).

$$
\frac{d^{\alpha}}{d x^{\alpha}} f(x)=\frac{1}{\Gamma(n-\alpha)} \frac{d^{n}}{d x^{n}} \int_{a}^{x} \frac{f(\eta)}{(x-\eta)^{\alpha+1-n}} d \eta,
$$

where $n=\lceil\alpha\rceil$ is the integer such that $n-1<\alpha \leq n$.

Note that the integer cases $\alpha=1$ and 2 are the classical advective flow and diffusion equations respectively. The case of non-integer $\alpha$ is used to model the super diffusive or anomalous diffusive flow in which a cloud of particles spreads at a faster rate than in the classical model [5]-[8].

Different approximation schemes for fractional derivatives have been proposed in the recent past [9]-[12]. Due to its non-local nature-that is, the calculation of fractional derivative at a certain point requires function values far from the point as well-the fractional derivative has more complex expressions than its integer counterparts. Consequently, the approximation of the fractional derivative involves grid points up to a boundary of the domain of the function concerned.

One of the approximations for the fractional derivative is given by finite differences based on left sided Grünwald-Letnikov formulae [13]-[15] given in equation (3) in Section II.

The approximation presents some limitations. First, it results in unstable numerical methods for initial value problems even for some implicit methods that are well known to be stable for integer derivatives. Second, the order of accuracy for approximation of the fractional derivative is never more than one [16]. To remedy the instability, a shifted Grünwald approximation is used in some recent literatures [17]. Still, the order of error of the approximation remains the same -the first order [17]. This is considered a limitation in improving the accuracy of finite difference approximation of the Grünwald-Letnikov formulation[16].

In this paper, we claim that the approximation based on the Grünwald-Letnikov formulation is, in fact, of second order accuracy for the fractional derivative at an intermediate point between the nodal points.

We use this fact to solve the fractional diffusion (1) and show that it gives an unconditionally stable method of second order accuracy for the solution with a Crank-Nicolson type formulation.

Earlier, second order accuracy for the fractional diffusion equation was obtained in [17] by first computing the Crank-Nicolson method for two uniform grid partitions, giving two approximate solutions of first order accuracy for the spatial dimension, and then by extrapolating the two results to obtain the second order accuracy. The number of grid points in the second partition is double of the first. In our approach, we directly obtain the second order accuracy from 
only one grid partition which is coercer of the two considered by them.

In Section II we demonstrated the second order accuracy of the Grünwald approximation. In Section III we justified the second order accuracy of the approximation. In Section IV we derived a Crank-Nicolson type method for the approximating the fractional diffusion equation. In Section $\mathrm{V}$ we proved the stability of the approximation methods. In Section VI we presented some numerical results to verify our claim.

\section{FraCtionAl Derivative AND APPROXIMATION}

Let a function $f(x)$ be defined in a bounded interval $[a, b]$ and be sufficiently differentiable to satisfy the results that follow. We assume that $f(x)=0$ for $x \leq a$.

For a real number $\alpha \in R$, the fractional derivative $d^{\alpha} f(x) / d x^{\alpha}$ is defined, among many formulations, by the standard Grünwald-Letnikov formulation

$$
{ }_{a} D^{\alpha} f(x):=\frac{d^{\alpha}}{d x^{\alpha}} f(x)=\lim _{h \rightarrow 0} \frac{1}{h^{\alpha}} \sum_{k=0}^{N(x)}(-1)^{k}\left(\begin{array}{l}
\alpha \\
k
\end{array}\right) f(x-k h)
$$

where $N(x) \rightarrow \infty$ as $h \rightarrow \infty$ and $\left(\begin{array}{l}\alpha \\ k\end{array}\right)=\frac{\Gamma(\alpha+1)}{\Gamma(k+1) \Gamma(\alpha-k+1)}$. The upper limit $N(x)$ of the index of summation is often taken as $N(x)=\lfloor(x-a) / h\rfloor$, where $\lfloor x\rfloor$ is the floor integer of $x$, but, it could be anything diverging as $h$ approaches zero. Another choice of $N(x)$ for efficient computation is considered in [18]. The formulation (2) stems from the formulation of the integer order derivatives extended to a real number. For a review of Grünwald-Letnikov and various other formulations and examples, see [19] and the references therein.

For a fixed $h$, the fractional derivative is approximated by simply dropping the limit in the Grünwald-Letnikov formulae as

$$
\delta_{x}^{\alpha} f(x)=\frac{1}{h^{\alpha}} \sum_{k=0}^{N(x)} \omega_{\alpha, k} f(x-k h)
$$

where $\omega_{\alpha, k}=(-1)^{k}\left(\begin{array}{l}\alpha \\ k\end{array}\right)$. We refer $\delta_{x}^{\alpha} f(x)$ the Grünwald approximation of ${ }_{a} D^{\alpha} f(x)$. The coefficients $\omega_{\alpha, k}$ can be computed by the recursive formulae $\omega_{\alpha, 0}=1$, $\omega_{\alpha, k+1}=\omega_{\alpha, k}(k-\alpha) /(k+1)$.

In Tadjeran et al.[17], it is proved that the approximation (3) is of first order accuracy. Earlier, Lubich [12] also showed the first order accuracy of this approximation. Subsequently, many researchers have considered this first order accuracy as a fact for the Grünwald approximation (3). (See also, for instance, [16]).

Moreover, the approximation (3) fails to give stable solutions, even for traditionally stable methods, such as implicit and Crank-Nicolson methods. In [17], a shifted version of the Grünwald approximation was considered with an 'integral' right shift of the grid points of the approximating domain to gain the stability back. In other words, (3) is considered an approximation of the fractional derivative at an inner grid point $x-p h$, where $p$ is an integer, instead of the right most grid point $x$. For $1<\alpha \leq 2$, the optimal choice for $p$ is one $(p=1)$. Still, the order of the approximation remains the same - the first order. We state below the result obtained in [17].

Proposition 2. 1. Let $1 \leq \alpha \leq 2, f \in C^{n+3}(R)$ such that all derivatives of $\mathrm{f}$ up to order $\mathrm{n}+3$ belong to $L^{1}(R)$. For any integer $p \geq 0$, define the shifted Grünwald difference by

$$
\delta_{x, p}^{\alpha} f(x)=\frac{1}{h^{\alpha}} \sum_{j=0}^{N(x)} \omega_{\alpha, j} f(x-(j-p) h)
$$

Then, we have, for some constants $a_{l}$ independent of $h, f$ and $x$,

$$
{ }_{-\infty} D^{\alpha} f(x)-\delta_{x, p}^{\alpha} f(x)=\sum_{l=1}^{n-1} a_{l-\infty} D^{\alpha+l} f(x) h^{l}+O\left(h^{n}\right)
$$

uniformly in $x \in R$.

When the function $f(x)$ is extended leftward with zero values, the location of the left boundary $a<x_{L}$ in the definition becomes unimportant. Therefore, $a=-\infty$ in (5) is not a problem.

The first two coefficients in (5), $a_{1}=\alpha / 2-p$ and $a_{2}=-1 / 2(p-\alpha / 2)^{2}-\alpha / 24$, are of interest to us.

Since the shift $p$ is an integer, while $1<\alpha \leq 2$, we have $a_{1} \neq 0$. This means that (4) is a first order approximation of the fractional derivative and never improves as long as $p$ remains an integer.

However, if we allow $p$ to have any real value, for $1 \leq \alpha \leq 2$, one may choose $p=\alpha / 2$ and therefore force $a_{1}$ to vanish resulting a second order approximation by (4) with $a_{2}=-\alpha / 24$. That is, $\delta_{x, \alpha / 2}^{\alpha} f(x)$ will give a second order approximation to the fractional derivative (2).

Note that, when $\alpha=1$, this will mean the second order two point central difference formulae for the derivative at the mid point with $p=1 / 2$,

$$
f^{\prime}(x)=(f(x+h / 2)-f(x-h / 2)) / h+O\left(h^{2}\right),
$$

and when $\alpha=2$, the second order three point central difference formulae for $f^{\prime \prime}(x)$ at the mid grid point with $p=1$,

$$
f^{\prime \prime}(x)=(f(x+h)-2 f(x)+f(x-h)) / h^{2}+O\left(h^{2}\right) .
$$

The fractional shift may, therefore, be considered a 
generalization of the above two cases to the fractional derivative.

Of course, this brings a misalignment of the grid points to intermediate points between the equi-spaced grid points. This misalignment can be avoided by shifting the point of the derivative to an intermediate point thus realigning the points of the difference formulae back to the grid points as shown in Fig. 1 .

\section{Grid points}

Grunwald unshifted

Grunwald shifted ( $p=1$ )

Grunwald shifted ( $p=\alpha / 2$ )

Re-aligned

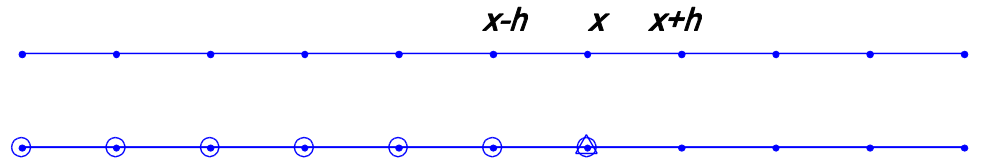

$\odot$

$\odot$

$\odot \quad$

$\odot$

$\odot$

( )

Fig. 1. Alignment and realignment of the grid points due to fractional shift.

\section{A SECOND ORDER APPROXIMATION}

In this section we give a mathematically rigorous setting for the second order approximation discussed in Section II.

Theorem 3.1. Let $f \in C^{n+3}(R)$ such that $f^{(k)} \in L^{1}(R)$ for all $k \leq n+3$. For a fixed $\mathrm{h}$, and for a parameter $\beta$, there exist coefficients $a_{l}(\beta)$, independent of $\mathrm{h}, \mathrm{f}$ and $\mathrm{x}$, but dependent on $\beta$ such that

$$
\begin{aligned}
{ }_{-\infty} D^{\alpha} f(x+\beta h) & -\delta_{x, p}^{\alpha} f(x) \\
& =\sum_{l=1}^{n-1} a_{l}(\beta)_{-\infty} D^{\alpha+l} f(x) h^{l}+O\left(h^{n}\right)
\end{aligned}
$$

Here, $p$ is an integer. When $\beta=p-\alpha / 2$, the approximation has an error of $O\left(h^{2}\right)$.

Proof: The proof closely follows the result of [17]. Therefore, we skip the details which are replicate in their proof.

The Fourier transform of a function $f(x)$ is given by $F[f(x)](k)=\hat{f}(k)=\int_{R} e^{i k x} f(x) d x . \quad$ It $\quad$ is known that $F[f(x+\beta h)](k)=e^{-i k \beta h} \hat{f}(k)$ and

$F\left[D^{\alpha} f(x)\right](k)=(-i k)^{\alpha} \hat{f}(k)$. (see also [19]).

Now, taking Fourier transform of the left hand side of (6),

$$
\begin{aligned}
F\left[_{-\infty}\right. & \left.D^{\alpha} f(x+\beta h)-\delta_{x, p}^{\alpha} f(x)\right](k) \\
& =\left((-i k)^{\alpha} e^{-i k \beta h}-\frac{e^{-i k p h}}{h^{\alpha}} \sum_{j=0}^{\infty} \omega_{\alpha, j} e^{-i k j h}\right) \hat{f}(k) \\
& =\left(e^{\beta z}-e^{p z}\left(\frac{1-e^{-z}}{z}\right)^{\alpha}\right)(-i k)^{\alpha} \hat{f}(k)=: g(z)(-i k)^{\alpha} \hat{f}(k),
\end{aligned}
$$

where $z=-i k h$. The function $g(z)$, being analytic, has an expansion $g(z)=\sum_{l=0}^{\infty} a_{l} z^{l}$, where $a_{l}$ are dependent on $\alpha, \beta$ and $p$ only. Therefore, with the conditions of the theorem, the analysis in [17] concludes (6).

In the expansion of $g(z)$, we have the coefficients $a_{0}=0, \quad a_{1}=\beta-\left(p-\frac{\alpha}{2}\right)$, and $a_{2}=-\frac{\alpha}{24}-\frac{1}{2}\left(p-\frac{\alpha}{2}\right)^{2}+\frac{\beta^{2}}{2}$. When $\beta=p-\frac{\alpha}{2}$, we have an estimate of order 2 with $a_{2}=-\frac{\alpha}{24}$.

Remark 3.1. By the choice of the parameter $\beta$, which depends on the integer shift, the arbitrary right shift $p$ becomes irrelevant to the formula. For $1 \leq \alpha \leq 2$, a shift with $p=1$ gives $\beta=1-\alpha / 2$ and for the un-shifted formula with $p=0$, we have $\beta=-\alpha / 2$. Thus, the derivative point for the second order approximation is always between the two rightmost grid points $x$ and $x+h$. (Fig. 1).

Remark 3.2. For $\alpha=1$, we get the second order two point central difference formulae in the form

$$
f^{\prime}(x+h / 2)=\frac{f(x+h)-f(x)}{h}+O\left(h^{2}\right)
$$

and when $\alpha=2$, we have the second order central three point formulae for the second derivative at $x$.

$$
f^{\prime \prime}(x+h)=[f(x+2 h)-2 f(x+h)+f(x)] / h^{2}+O\left(h^{2}\right) .
$$

\section{APPLICATION TO FRACTIONAL DIFFUSION EQUATION}

We derive a Crank-Nicolson type scheme for the fractional diffusion (1) and show that it is consistent with second order accuracy. We assume that the fractional differential (1) with the given initial and boundary conditions is well-posed, having a unique solution.

For the finite difference approximation of (1), the spatial domain $\left[x_{L}, x_{R}\right]$ is discretized into $N$ uniform subintervals of size $\Delta x=\left(x_{R}-x_{L}\right) / N$. The time domain dscretization 
with $M$ subintervals of size $\Delta x=T / M$ is used for the interval $0 \leq t \leq T$. Denote the spatial and time grid points as $x_{i}=x_{L}+i \Delta x, \quad i=0,1, \cdots, N$ and $t_{j}=j \Delta t, \quad j=0,1, \cdots, M$ respectively.

In order to apply the second order approximations of the spatial fractional derivative and a second order approximation for the time derivative, the diffusion (1) is expressed in a shifted form in both spatial and time variables as

$$
\begin{aligned}
D_{t} u(x+ & \beta \Delta x, t+\Delta t / 2) \\
= & d(x+\beta \Delta x) D_{x}^{\alpha} u(x+\beta \Delta x, t+\Delta t / 2) \\
& +q(x+\beta \Delta x, t+\Delta t / 2),
\end{aligned}
$$

where $D_{t}=\partial / \partial t$ and $D_{x}^{\alpha}=\partial^{\alpha} / \partial x^{\alpha}$.

For the approximation of function values at shifted points by the function values at the grid points, we have the following.

Proposition 4.1. Let $f \in C^{2}(R)$ be a function having derivatives up to order 2 . For a fixed $h>0$ and $\eta \in R$, we have for the function value $f(x+\eta h)$ :

$$
f(x+\eta h)=(1-\eta) f(x)+\eta f(x+h)+O\left(h^{2}\right) .
$$

Proof. Taylor series expansions.

The fractional derivative at the shifted point $x+\beta \Delta x$ in (8) is approximated by the difference approximation $\delta_{x, 1}^{\alpha} f(x)$ in (4) (with $p=1$ ) and the time derivative at $t+\Delta t / 2$ is approximated by the second order central difference approximation in (7). This gives

$$
\begin{aligned}
& \frac{u(x+\beta \Delta x, t+\Delta t)-u(x+\beta \Delta x, t)}{\Delta t} \\
& =d(x+\beta \Delta x) \delta_{x, 1}^{\alpha} u(x, t+\Delta t / 2)+q(x+\beta \Delta x, t+\Delta t / 2) \\
& \quad+a_{1} C \Delta x+O\left(\Delta x^{2}\right)+O\left(\Delta t^{2}\right) .
\end{aligned}
$$

Here, we separated the coefficient factor $a_{1}=\beta-(1-\alpha / 2)$ for later analysis.

Again, the function value of $u(x+\beta \Delta x, \cdot)$ is approximated by the second order forward approximation in equation (9). Moreover, the function value of $u(\cdot, t+\Delta t / 2)$ in the approximation of fractional derivative is approximated by the second order approximation for the time in equation (9) with $\eta=1 / 2$. These are the averages of the adjacent function values of the time grids $t+\Delta t$ and $t$, and results in the Crank-Nicolson type method. Altogether, these give

$$
\begin{aligned}
& \beta u(x+\Delta x, t+\Delta t)+(1-\beta) u(x, t+\Delta t) \\
& -(\beta u(x+\Delta x, t)+(1-\beta) u(x, t) \\
& \quad=d(x+\beta \Delta x) / 2\left(\delta_{x, 1}^{\alpha} u(x, t+\Delta t)+\delta_{x, 1}^{\alpha} u(x, t)\right) \\
& \quad+q(x+\beta \Delta x, t+\Delta t / 2)+a_{1} C \Delta x+O\left(\Delta x^{2}\right)+O\left(\Delta t^{2}\right) .
\end{aligned}
$$

Denoting $\quad u\left(x_{i}, t_{n}\right):=u_{i}^{n}, d_{i+\beta}:=d\left(x_{i}+\beta \Delta x\right) \quad$ and $q_{i+\beta}^{n+1 / 2}:=q(x+\beta \Delta x, t+\Delta t / 2), \quad$ the second order approximation for both spatial and time variables is given by

$$
\begin{aligned}
(1-\beta) & \frac{u_{i}^{n+1}-u_{i}^{n}}{\Delta t}+(1-\beta) \frac{u_{i+1}^{n+1}-u_{i+1}^{n}}{\Delta t} \\
= & \frac{d_{i+\beta}}{2}\left(\delta_{x, 1}^{\alpha} u_{i}^{n+1}+\delta_{x, 1}^{\alpha} u_{i}^{n}\right)+q_{i+\beta}^{n+1 / 2}+a_{1} C \Delta x \\
& +O\left(\Delta x^{2}\right)+O\left(\Delta t^{2}\right) .
\end{aligned}
$$

When $\beta=1-\alpha / 2$, we have $a_{1}=0$ and obtain the second order approximation. For other choices it remains at first order.

We denote by $U_{i}^{n}$ the numerical approximation of the function values $u_{i}^{n}$. The Crank-Nicolson type approximation of the fractional diffusion equation is then given by

$$
\begin{aligned}
(1-\beta) & U_{i}^{n+1}+\beta U_{i+1}^{n+1}-\left((1-\beta) U_{i}^{n}+\beta U_{i+1}^{n}\right) \\
= & \frac{d_{i+\beta} \Delta t}{2 \Delta x^{\alpha}}\left(\sum_{k=0}^{i+1} \omega_{\alpha, k} U_{i-k+1}^{n+1}+\sum_{k=0}^{i+1} \omega_{\alpha, k} U_{i-k+1}^{n}\right) \\
& +\Delta t q_{i+\beta}^{n+1 / 2}, i=0,1,2, \cdots, N-1 ; n=0,1, \cdots, M-1 .
\end{aligned}
$$

Defining the column vectors $\mathbf{U}^{n}=\left[U_{1}^{n}, U_{2}^{n}, \cdots, U_{N-1}^{n}\right]^{T}$,

$\mathbf{q}_{\beta}^{n+1 / 2}=\Delta t\left[q_{1}^{n+1 / 2}, q_{2}^{n+1 / 2}, \cdots, q_{N-1}^{n+1 / 2}\right]^{T}$ and

$\eta_{i+\beta}=d_{i+\beta} \Delta t /\left(2 \Delta x^{\alpha}\right)$, the equation (10) may be written in matrix form as

$$
\mathbf{T}_{\beta}^{F} \mathbf{U}^{n+1}-\mathbf{T}_{\beta}^{F} \mathbf{U}^{n}=\mathbf{A}_{\beta}\left(\mathbf{U}^{n+1}+\mathbf{U}^{n}\right)+\mathbf{q}_{\beta}^{n+1 / 2}+\mathbf{b}_{\beta}^{F},
$$

where $\mathbf{T}_{\beta}^{F}, \mathbf{A}_{\beta}$ are Toeplitz matrices and $\mathbf{b}_{\beta}^{F}$ is the vector obtained by imposing the boundary conditions. They are given by

$$
\begin{gathered}
\mathbf{T}_{\beta}^{F}(i, j)=\left\{\begin{array}{cc}
1-\beta, & j=i \\
\beta, & j=i+1, \\
0, & \mathrm{e} / \mathrm{w}
\end{array}\right. \\
\mathbf{A}_{\beta}(i, j)=\left\{\begin{array}{cc}
\eta_{i+\beta} \omega_{\alpha, i-j+1}, & j \leq i+1 \\
0, & j>i,
\end{array}\right. \text { and }
\end{gathered}
$$

Rearranging (11), we obtain the iterative scheme

$$
\left(\mathbf{T}_{\beta}^{F}-\mathbf{A}_{\beta}\right) \mathbf{U}^{n+1}=\left(\mathbf{T}_{\beta}^{F}+\mathbf{A}_{\beta}\right) \mathbf{U}^{n}+\mathbf{q}_{\beta}^{n+1 / 2}+\mathbf{b}_{\beta}^{F},
$$

One may solve (12) to obtain the approximate solution $\mathbf{U}^{n+1}$ at the time step $t_{n+1}$ with initial condition $\mathbf{U}^{0}=\left[s_{0}\left(x_{1}\right), s_{0}\left(x_{2}\right), \cdots, s_{0}\left(x_{N-1}\right)\right]$. 
Remark 4.1. For $\beta=0$, we have the first order $\mathrm{CN}$ formulation given in [17]. For $\beta=1-\alpha / 2$, we obtain a Crank-Nicolson type formulations with second order accuracy. Also note that, by choosing $\beta=1$, we get the first order 'un-shifted' Crank-Nicolson formulation which is surprisingly unstable [13].

Moreover, for $\alpha=2$, we have $\beta=1-\alpha / 2=0$, and get the classical Crank-Nicolson formulation of order 2 , shifted by default.

Remark 4.2. Since the system to be solved involves only Toeplitz matrices, a fast algorithm using FFT can be employed by doubling the size of the system. For details of this approach for a similar system, see [18].

We compare the computational cost of our method with what was given in [17]. Note that the method given in [17] with extrapolation to obtain second order accuracy used three times the number of the grid points used in our approach. If a naïve (direct) solver of $O\left(N^{2}\right)$ is applied to the system (10) with operation count $K N^{2}$, where $K$ is a suitable constant, the saving ratio of operations count relative to our method is $K(3 N)^{2} /\left(K N^{2}\right)=9$ which has relative saving of $(9-1) / 9=88.88 \%$. On the other hand, if the FFT approach with $O\left(N \log _{2} N\right)$ given in [18] is applied to both methods, the ratio of operations count will be $K 3 N \log _{2} 3 N /\left(K N \log _{2} N\right) \geq 3$, which has a minimum saving of $(3-1) / 3=66.66 \%$.

\section{Stability AND CONVERGEnCE}

As for the stability of the scheme (12), we have the following result.

Theorem 5. 1. The Forward Crank-Nicolson scheme (12) is stable unconditionally of the choice of the grid points if and only if $\beta \leq 1 / 2$.

Proof: We shall prove that the magnitude of the eigenvalues of the iterative matrix $\left(T_{\beta}^{F}-A_{\beta}\right)^{-1}\left(T_{\beta}^{F}+A_{\beta}\right)$ is $<1$.

For this, we have the following steps:

1) The real parts of the eigenvalues of $A_{\beta}$ are negative and they are characterized by the symmetric part $A_{\beta}^{S}=\left(A_{\beta}+A_{\beta}^{T}\right) / 2$ of $A_{\beta}$. For, by Greschgorin theorem [21], the eigenvalues of a matrix $A$ are in the disks centered at each diagonal entry $C_{i}=A_{i, i}$ with radius $r_{i}=\sum_{k=1, k \neq i}^{n}\left|A_{i, k}\right|$. For the matrix $A_{\beta}$, we have

$C_{i}=\eta_{i+\beta} w_{\alpha, 1}=-\eta_{i+\beta} \alpha$ and

$r_{i}=\eta_{i+\beta} \sum_{k=1, k \neq i}^{i+1} w_{\alpha, i-k+1}<\eta_{i+\beta} \alpha$. Therefore, these disks

lie in the left half of the complex plane and thus the real parts of the eigenvalues are negative.

2) The matrix $T_{\beta}^{F}$ is positive definite if, and only if, $\beta<1 / 2$. It is clear since $|1-\beta|>|\beta|$ iff $\beta<1 / 2$. The matrix also has a real eigenvalue $1-\beta$ of multiplicity $N-1$.

3) The eigenvalues of $G=\left(T_{\beta}^{F}\right)^{-1} A_{\beta}$ have negative real parts. For, the real part of the eigenvalues of $G$ will lie in $m \leq\left(x^{T} A_{\beta}^{S} x\right) /\left(x^{T} T_{\beta}^{F} x\right) \leq M$. where $m, M$ are the minimum and maximum of the eigenvalues. As $T_{\beta}^{F}$ is positive definite, we have $m \leq M<0$ by i.

4) Since $C=\left(T_{\beta}^{F}-A_{\beta}\right)^{-1}\left(T_{\beta}^{F}+A_{\beta}\right)=(I-G)^{-1}(I+G)$, if $\lambda$ is an eigenvalue of $G$, then $(1-\lambda) /(1+\lambda)$ is an eigenvalue of $C$. We have $\operatorname{Re}(\lambda)<0$, and therefore $|(1-\lambda) /(1+\lambda)|<1$ provided that $\beta<1 / 2$.

Now, for our fractional diffusion equation, we have $\beta=1-\alpha / 2$ with $1<\alpha \leq 2$. So, $0 \leq \beta<1 / 2$, and hence the forward $\mathrm{C}-\mathrm{N}$ approximation is unconditionally stable.

Now, the following cases can be considered for stability analysis (see also Remark 4.1):

In the FCN formulation,

1) For $1 \leq \alpha \leq 2$, the choice $\beta=1-\alpha / 2$ satisfies $0 \leq \beta \leq 1 / 2$. Therefore, our proposed second order FCN approximation is unconditionally stable.

2) The choice $\beta=0$ gives the shifted Crank-Nicolson type approximation which is unconditionally stable also proved in [13], [17].

3) When $\beta=1$ we get the un-shifted Crank-Nicolson approximation which is unstable violating the condition of Theorem 5.1. This was demonstrated in [13].

With the consistency established in Section IV and the unconditional stability, we conclude by the Lax's equivalence theorem that the FCN approximation methods for the fractional diffusion (1) are convergent.

\section{NUMERICAL TEST}

For testing the method proposed in this paper, we consider the problem in [17] given with the following data:

$$
\frac{\partial}{\partial t} u(x, t)=d(x) \frac{\partial^{\alpha}}{\partial x^{\alpha}} u(x, t)+q(x, t), \quad 0 \leq x \leq 1, t \geq 0,
$$

$u(0, t)=0, u(1, t)=b_{R}(t), t>0, \quad u(x, 0)=s_{0}(x), \quad 0<x<1$.

with

Fractional derivative order $\alpha=1.8$,

diffusion coefficient $d(x)=\Gamma(2.2) x^{2.8} / 6$,

the source function $q(x, t)=-(1+x) x^{3} \exp (-t)$,

the initial condition $u(x, 0)=s_{0}(x)=x^{3}, \quad 0 \leq x \leq 1$

and the boundary conditions

$$
u(0, t)=0, \quad u(1, t)=b_{R}(t)=\exp (-t), t>0 .
$$

The analytical solution to the problem is known to be $u(x, t)=x^{3} \exp (-t)$ (see also [20]).

We computed the solution for the case $\beta=0$ which gives 
the first order stable method obtained in [13] and for the case $\beta=0.1=1-\alpha / 2$ which gives the proposed second order stable method. The grid subinterval sizes were chosen as $\Delta x=\Delta t=1 / 10,1 / 15 /, 1 / 20,1 / 25,1 / 100,1 / 500$ and $1 / 1000$. Tables 1 shows the absolute errors at time $t=1$. Note that the results of the $\mathrm{CN}$ formulation are the same obtained in [17]. Fig. 2 is the log-log plot of the errors. The convergence order of the log-log plot of error is computed as follows:

Let $E_{i}=\max _{0<k<N}\left|u_{k}^{M}-U_{k}^{M}\right|$ be the maximum absolute error for the partition size at row $i$ in Tables I. The order of the error will be given by the slope of the log-log graph. The slope for the successive points are given by

$$
\begin{aligned}
m_{i+1} & =\left(\log E_{i+1}-\log E_{i}\right) /\left(\left(\log x_{i+1}-\log x_{i}\right)\right. \\
& =\log \left(E_{i+1} / E_{i}\right) / \log \left(x_{i+1} / x_{i}\right) .
\end{aligned}
$$

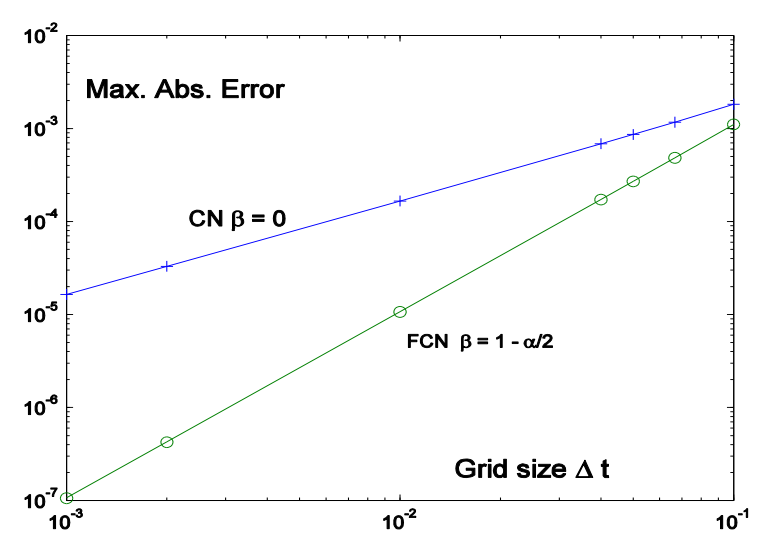

Fig. 2. Log-log plot for CN, FCN.

We also computed the solution with $\beta=1$. Theoretically, it represents the unstable $\mathrm{CN}$ formulation. Numerical results also displayed unstable behavior in the solution.

\begin{tabular}{|c|c|c|c|c|c|}
\hline \multirow{2}{*}{$i$} & \multirow{2}{*}{$\Delta x=\Delta t$} & \multicolumn{2}{|c|}{$\beta=0$} & \multicolumn{2}{|c|}{$\mathrm{FCN}(\beta=1-\alpha / 2)$} \\
\hline & & Error $E_{i}$ & Order & Error $E_{i}$ & Order \\
\hline 1 & $1 / 10$ & $1.8226 \mathrm{e}-3$ & -- & $1.1045 \mathrm{e}-3$ & -- \\
\hline 2 & $1 / 15$ & $1.1681 \mathrm{e}-3$ & 1.0972 & $4.8323 \mathrm{e}-4$ & 2.0388 \\
\hline 3 & $1 / 20$ & $8.6482 \mathrm{e}-4$ & 1.0449 & $2.6981 \mathrm{e}-4$ & 2.0258 \\
\hline 4 & $1 / 25$ & $6.8495 \mathrm{e}-4$ & 1.0450 & $1.7193 \mathrm{e}-4$ & 2.0193 \\
\hline 5 & $1 / 100$ & $1.6597 \mathrm{e}-4$ & 1.0225 & $1.0644 \mathrm{e}-5$ & 2.0069 \\
\hline 6 & $1 / 500$ & $3.2911 \mathrm{e}-5$ & 1.0053 & $4.2447 \mathrm{e}-7$ & 2.0019 \\
\hline 7 & $1 / 1000$ & $1.6438 \mathrm{e}-5$ & 1.0015 & $1.0608 \mathrm{e}-7$ & 2.0005 \\
\hline
\end{tabular}

TABLE I. ABSOLUTE ERROR AND CONVERGENCE ORDER FOR FCN

\section{CONCLUSION}

We have considered the finite difference approximation of fractional diffusion equation. The well known Grünwald approximation of the fractional derivative is shown to have second order accuracy at an intermediate point between two adjacent grid points. This fact is used to device second order Crank-Nicolson type formulations for the fractional diffusion equations. This formulation is a general in the sense that it covers three cases of formulations in one setting.

\section{REFERENCES}

[1] D. A. Benson, S. W. Wheatcraft, and M. M. Meerschaert, "Application of fractional advection-dispersion equation," Water Resour. Res., vol. 36, pp. 1403-1412, 2000.

[2] R. Metzler and J. Klafter, "Accelerating Brownian motion: A fractional dynamics approach to fast diffusion," Europhys. Lett., vol. 51, pp. 492-498, 2000.

[3] J. A. T. Machado, "Fractional derivatives: Probability interpretation and frequency response of rational approximations," Commun Nonlinear Sci Numer Simulation, vol. 14, pp. 3492-3497, 2009.

[4] R. Metzler and J. Klafter, "The random walk's guide to anomalous diffusion: A fractional dynamics approach," J. Contam., Hydrol., vol. 85, pp. 53-71, 2000.

[5] B. Baeumer, M. Kovacs, and M. M. Meerchaert, "Numerical solutions for fractional reaction-diffusion equations," Comput. Math. Appl., vol. 55, pp. 2212-2226, 2008.

[6] R. Schumer, M. M. Meerchaert, and B. Baeumer, "Fractional advection-dispersion equations for modeling transport at the earth surface," J. Geophys. Res., vol. 114, doi:10.1029/2008JF001246, 2009.

[7] L. Zhou and H. M. Selim, "Application of the fractional advection-dispersion equation in porous media," Soil. Sci. Soc. Am. J., vol. 67, pp. 1079-1084, 2003.

[8] I. Podlubny, Fractional differential equations, New York: Academic Press, Ch. 2, 1999.

[9] J. Frank, W. Hundsdorfer, and J. G. Verwer, "On the stability of implicit-explicit linear multistep methods," Applied Numerical Mathematics, vol. 25, pp. 193-205, 1997.

[10] R. Garrappa, "On some explicit Adams multistep methods for fractional differential equations," J. Comput. Appl. Math, vol. 229, pp. 392-399, 2009.

[11] T. A. M. Langlands and B. I. Henry, "The accuracy and stability of an implicit solution method for the fractional diffusion equation," $J$. Comput. Phys., vol. 205 pp. 719-736, 2005.

[12] C. Lubich, "Discretized fractional calculus," SIAM J. MATH. ANAL. vol. 17 , no. 3 , pp. 704-719, 1986.

[13] M. M. Meerchaert and C. Tadjedran, "Finite difference approximations for fractional-advection-dispersion flow equations," J. Comp. Appl. Math., vol. 172, pp. 65-77, 2004.

[14] J. P. Roop, "Computational aspects of FEM approximation of fractional advection dispersion equations on bounded domains in $\mathrm{R}^{2}$," $J$. Comp. Appl. Math., vol. 193, pp. 243-268, 2006.

[15] E. Sausa, "Finite difference approximations for a fractional advection diffusion problem," J. Comput. Phys., vol. 228, pp. 4038-4054, 2009.

[16] E. Sausa, "How to approximate the fractional derivative of order $1<\alpha \leq 2$," in Proc. FDA'10, I. Podlubny et al., Eds, Article No. FDA10-019, 2010.

[17] C. Tadjedran, M. M. Meerchaert, and H. Scheffler, "A second order accurate numerical approximation for the fractional diffusion equation," J. Comput. Phys., vol. 213, pp. 205-213, 2006.

[18] H. Wang, K. Wang, and T. Sircar, "A direct O ( $\left.\operatorname{Nog}_{2} \mathrm{~N}\right)$ finite difference method for fractional diffusion equations," J. Comput. Phys., vol. 229, pp. 8095-8104, 2010.

[19] G. Dahlquist, "Convergence and stability in the numerical integration of ordinary differential equations," Mathematical Scandinavia, vol. 4 , pp. 33-53, 1956.

[20] S. Saha Ray, "Analytical solution for the space fractional diffusion equation by two-step adomain decomposition method," Communications in Nonlinear Science and Numerical Simulation, vol. 14, pp. 1295-1306, 2009.

[21] E. Isaacson and H. B. Keller, Analysis of numerical methods, New York: Wiley, 1966.

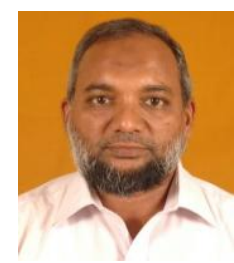

Haniffa M. Nasir was born in Sri Lanka on 16th November, 1962. He obtained his B.Sc. degree in mathematics from University of Jaffna, Sri Lanka and obtained his M.Sc. and Ph.D. degrees in numerical analysis of Wave Scattering problems from The University of Electro-Communications, Tokyo, Japan. This author became a Senior Member(SM) of IACSIT. He was Lecturer in University of Jaffna, Sri Lanka, Senior Lecturer in University of Peradeniya. He served as Head of Department since 2010. Currently, he is a Visiting Faculty in the Department of Mathematics and Statistics, Sultan Qaboos University, Al-Khoud 123, Muscat, Sultanate of Oman. Dr. Nasir is also member of the Sri Lanka Association for Advancement of Science(SLAAS), the Senior Scientist Forum (SSF) in Sri Lanka and Japan Society of Industrial and Applied Mathematics (JSIAM). 


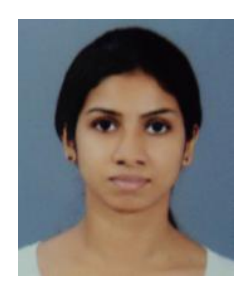

Lakhshika K. Gunawardana was born in Sri Lanka on 9th March, 1987. She obtained her B.Sc. degree in mathematics from University of Peradeniya, Sri Lanka in 2010. She is currently attached with the Department of Mathematics, University of Peradeniya, Sri Lanka as lecturer.

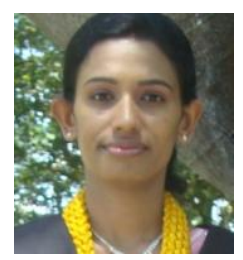

Niroshani P. Abeyrathna was born in Sri Lanka on 21st April, 1986. She obtained her B.Sc. degree in mathematics from University of Peradeniya, Sri Lanka in 2010. She is currently attached with the Department of Mathematics, University of Peradeniya, Sri Lanka as lecturer . 